\title{
FKBP5 Moderates Alcohol Withdrawal Severity: Human Genetic Association and Functional Validation in Knockout Mice
}

\author{
Ming-Chyi Huang ${ }^{1,2}$, Melanie L Schwandt ${ }^{3}$, Julia A Chester ${ }^{4,5}$, Aaron M Kirchhoff ${ }^{4,5}$, Chung-Feng Kao ${ }^{6}$, \\ Tiebing Liang, ${ }^{4,5}$, Jenica D Tapocik ${ }^{3}$, Vijay A Ramchandani ${ }^{3}$, David T George ${ }^{3}$, Colin A Hodgkinson ${ }^{7}$, \\ David Goldman ${ }^{7}$ and Markus Heilig*,3
}

'Department of Psychiatry, Taipei City Psychiatric Center, Taipei City Hospital, Taipei, Taiwan; ${ }^{2}$ Department of Psychiatry, School of Medicine, Taipei Medical University, Taipei, Taiwan; ${ }^{3}$ Laboratory of Clinical and Translational Studies, National Institute on Alcohol Abuse and Alcoholism, National Institutes of Health, Bethesda, MD, USA; ${ }^{4}$ Department of Psychological Sciences, Purdue University, West Lafayette, IN, USA; ${ }^{5}$ Department of Medicine, Indiana University, School of Medicine, Indianapolis, IN, USA; ${ }^{6}$ Department of Public Health and Institute of Epidemiology and Preventive Medicine, College of Public Health, Taipei, Taiwan; ' Laboratory of Neurogenetics, National Institute on Alcohol Abuse and Alcoholism/National Institutes of Health, Bethesda, MD, USA

\begin{abstract}
Alcohol withdrawal is associated with hypothalamic-pituitary-adrenal (HPA) axis dysfunction. The FKBP5 gene codes for a cochaperone, FK506-binding protein 5, that exerts negative feedback on HPA axis function. This study aimed to examine the effects of single-nucleotide polymorphisms (SNPs) of the FKBP5 gene in humans and the effect of Fkbp5 gene deletion in mice on alcohol withdrawal severity. We genotyped six FKBP5 SNPs (rs3800373, rs9296158, rs3777747, rs9380524, rs I360780, and rs9470080) in 399 alcohol-dependent inpatients with alcohol consumption $48 \mathrm{~h}$ before admission and recorded scores from the Clinical Institute Withdrawal Assessment-Alcohol revised (CIWA-Ar). Fkbp5 gene knockout (KO) and wild-type (WT) mice were assessed for alcohol withdrawal using handling-induced convulsions ( $\mathrm{HICS}$ ) following both acute and chronic alcohol exposure. We found the minor alleles of rs3800373 (G), rs9296I58 (A), rs 1360780 (T), and rs9470080 (T) were significantly associated with lower CIWA-Ar scores whereas the minor alleles of rs3777747 (G) and rs9380524 (A) were associated with higher scores. The haplotype-based analyses also showed an association with alcohol withdrawal severity. Fkbp5 $\mathrm{KO}$ mice showed significantly greater HICs during withdrawal from chronic alcohol exposure compared with WT controls. This study is the first to show a genetic effect of FKBP5 on the severity of alcohol withdrawal syndrome. In mice, the absence of the Fkbp5 gene enhances sensitivity to alcohol withdrawal. We suggest that FKBP5 variants may trigger different adaptive changes in HPA axis regulation during alcohol withdrawal with concomitant effects on withdrawal severity.

Neuropsychopharmacology (20I4) 39, 2029-2038; doi:I0.I038/npp.20I4.55; published online 2 April 20I4
\end{abstract}

\section{INTRODUCTION}

The alcohol withdrawal syndrome (AWS) is precipitated by cessation of excessive alcohol consumption, and is characterized by symptoms of central nervous system hyperexcitability, autonomic hyperactivity, and tremor. These symptoms follow a characteristic temporal course, typically emerging within the first $24-48 \mathrm{~h}$ of abstinence and resolving within 1 week. Although neither necessary nor sufficient for a diagnosis of alcohol dependence (AD; hereafter equated with alcoholism), the AWS has recently received renewed research interest because of its potential

\footnotetext{
*Correspondence: Dr M Heilig, Laboratory of Clinical and Translational Studies, National Institute on Alcohol Abuse and Alcoholism, National Institutes of Health, Bethesda, MD 20892, USA, Tel: + I 3017687326 , Fax: + 30 I 45I 7498, E-mail: markus.heilig@mail.nih.gov Received 13 September 2013; revised 31 January 2014; accepted 25 February 2014; accepted article preview online 7 March 2014
}

role in long-term neuroadaptations that occur with repeated cycles of alcohol intoxication and withdrawal (Heilig et al, 2010). An understanding of genetic factors that moderate the severity of the AWS may therefore contribute to an improved understanding of neuroadaptive processes that contribute to the progressive course of alcoholism.

The intensity and time course of AWS varies between individuals, consistent with the possibility of underlying genetic influences, but inheritance of alcohol withdrawal severity has not been directly demonstrated in humans. Interestingly, a recent twin analysis indicated that the presence of withdrawal symptoms loads on a genetic factor that is also associated with the criterion of continued use despite problems (Kendler et al, 2012). In mice, alcohol withdrawal severity is moderately heritable (Metten and Crabbe, 2005). Nevertheless, genes influencing alcohol withdrawal severity are largely unknown, except for the multiple PDZ-binding domain protein (MPDZ), which may modulate withdrawal severity both in mice (Shirley et al, 
2004) and humans (Karpyak et al, 2009). Existing reports on the association of vulnerability genes with acute withdrawal severity have largely focused on specific severe phenotypes such as delirium tremens (DTs), seizures, or hallucinations (Okubo et al, 2003; Limosin et al, 2004; Rujescu et al, 2005; Tadic et al, 2005; Preuss et al, 2006; van Munster et al, 2007; Karpyak et al, 2010; Du et al, 2011), associations with high or low number of withdrawal symptoms (Schumann et al, 2003), or phenotypes categorized as mild or severe AWS (Schmidt and Sander, 2000; Koehnke et al, 2002; Schumann et al, 2003; Wernicke et al, 2003). However, the symptom profile of AWS runs on a continuum with substantial individual variation, and little is known about the potential genetic components that influence the severity of AWS in individuals who do not experience DTs or withdrawal seizures.

Hypothalamic-pituitary-adrenal (HPA) axis function involving glucocorticoid, central glucocorticoid receptors (GR), and corticotropin-releasing factor is dysregulated following chronic alcohol exposure. Alterations in HPA axis responsivity contribute to the emergence of withdrawal symptoms, which in turn influence the risk of relapse (Adinoff et al, 1998, 2005; Becker, 2012). Alcohol-dependent patients have increased cortisol levels despite blunted adrenocortical sensitivity during withdrawal (Costa et al, 1996; Adinoff et al, 1998; Esel et al, 2001) and the cortisol levels may be associated with relapse vulnerability (Stephens and Wand, 2012). Animal studies also have shown a considerable increase in both circulating and brain corticosterone levels following alcohol withdrawal (Rose et al, 2010) and elevated blood corticosterone levels could enhance alcohol withdrawal severity (Roberts et al, 1994) through GR activation (Sharrett-Field et al, 2013). These effects may be of interest beyond the acute withdrawal period, as recent evidence suggests a role for GR-mediated plasticity in escalation of voluntary alcohol consumption in rats (Vendruscolo et al, 2012).

Genetic factors that regulate GR signaling may contribute to individual differences in HPA axis responses. FK506binding protein 5 (FKBP5) is a co-chaperone of heat-shock protein 90 in an inactive GR complex. Once cortisol is bound to GR, FKBP5 is exchanged with other cochaperones, whereupon the GR complex can translocate to the nucleus and bind the DNA. The binding of FKBP5 to the GR complex causes lower affinity for cortisol and less efficient nuclear translocation of GR (Wochnik et al, 2005). Therefore, FKBP5 gene variants may influence GR sensitivity to cortisol and the efficiency of negative feedback on the HPA axis (Binder, 2009). Accordingly, variation in the FKBP5 gene has been associated with reduced dexamethasone suppression (Binder et al, 2008), incomplete normalization of stress-elicited cortisol secretion (Ising et al, 2008), and response to antidepressants and recurrence of depressive episodes (Binder et al, 2004). FKBP5 variation has also been shown to interact with childhood trauma exposure to increase the risk for posttraumatic stress disorder (PTSD) (Binder et al, 2008; Xie et al, 2010) as well as suicidal behavior (Roy et al, 2010). Furthermore, genotype-specific FKBP5 DNA methylation may mediate these genechildhood trauma interactions (Klengel et al, 2013). More importantly, a recent study has reported differential expression of the FKBP5 gene in postmortem brains of alcoholics (McClintick et al, 2013), suggesting a role for this gene in the pathophysiology of $\mathrm{AD}$. Here, we examined whether genetic variation at the FKBP5 locus moderates the severity of alcohol withdrawal in alcohol-dependent patients who had consumed their last drink within $48 \mathrm{~h}$ of admission to an inpatient unit. To functionally validate our human association findings, we assessed the withdrawal response following acute and chronic alcohol exposure in mice with deletion of the Fkbp5 gene.

\section{MATERIALS AND METHODS}

\section{Human Association Study}

Participants. Participants who met the Diagnostic and Statistical Manual for Mental Disorders, 4th Edition, Textrevised $(D S M-I V-T R)$ criteria for $\mathrm{AD}$ were consecutively recruited from September 2005 to March 2013 at the National Institute on Alcohol Abuse and Alcoholism (NIAAA) Inpatient Unit at the National Institutes of Health (NIH) Clinical Center where they were voluntarily admitted to a 28-day inpatient treatment protocol. Subjects had consumed their last alcoholic drink within $48 \mathrm{~h}$ of admission, were literate in English, and were not suffering from active psychotic symptoms or cognitive impairment. Informed consent was obtained in accordance with the Declaration of Helsinki and the NIH Combined NeuroScience Institutional review board.

Clinical assessment. The Structured Clinical Interview for DSM-IV Axis I Disorders (SCID) (First et al, 1996) was used for the diagnostic assessment of alcohol and other substance use disorders. Severity of AD was assessed using the Alcohol Dependence Scale (Skinner and Horn, 1984), and alcohol consumption during the preceding 3 months was assessed using the timeline follow-back (TLFB). Smoking intensity parameters included pack-years and Fagerstrom test of nicotine dependence score. The baseline measurement of depression and anxiety symptoms on the second day of admission were based on the Comprehensive Psychopathological Rating Scale (CPRS) (Asberg et al, 1978). Withdrawal intensity was evaluated using the Clinical Institute Withdrawal Assessment-Alcohol revised (CIWAAr) Scale approximately every $2-4 \mathrm{~h}$ while awake for the first 3-5 days after admission. Participants did not receive any prescription medications other than benzodiazepines (BZDs; diazepam, oxazepam, or lorazepam), which were given based on clinical decisions. We analyzed the maximum and average CIWA-Ar scores during withdrawal, as well as total BZD usage, which was converted to diazepam equivalent doses where necessary.

Genotyping. With the exception of rs1360780, genotyping was performed at the NIAAA Laboratory of Neurogenetics on the Illumina OmniExpress BeadChip (Illumina, San Diego, CA), which includes $>700000$ single-nucleotide polymorphisms (SNPs). The average genotype reproducibility was 0.99994 . Linkage disequilibrium (LD), that is, the non-random association of alleles at two or more loci, of the FKBP5 gene, is generally high in most populations including Caucasians and African Americans (Binder, 2009). In this study, the FKBP5 SNPs were selected based on those that 
have been implicated in a previous study (Roy et al, 2010) and could be captured from the available genetic information in the existing Illumina data set for FKBP5, including rs3800373, rs9296158, rs3777747, rs9380524, and rs9470080. An additional SNP not included in the Illumina OmniExpress array, rs1360780, was also genotyped as the functionality of this variant in the negative feedback of GR regulation has been investigated and characterized (Binder et al, 2004; Ising et al, 2008; Roy et al, 2010; Xie et al, 2010; Zannas and Binder, 2014). Rs1360780 was genotyped using the assay-on-demand (assay ID: C__8852038_10 ) from Applied Biosystems (Foster City, CA). The allele was discriminated by post-PCR plate read on ABI PRISM 7900HT Sequence Detector System (Applied Biosystems). Data were processed using SDS 2.4 software (Applied Biosystems) at the Laboratory of Clinical and Translational Studies, NIAAA, NIH. All six SNPs were in Hardy-Weinberg equilibrium (HWE) and had minor allele frequencies $>5 \%$.

Assessment of population stratification using ancestry informative markers. Ancestry informative markers (AIMs, $n=2500$ ) were extracted from the Illumina array to calculate ancestral proportions for all study participants (missing data for five subjects). Using methods described previously for an AIMs panel including 186 markers (Hodgkinson et al, 2008), which were not available for our data set, the ancestry assessment identified six ethnic factors (Africa, Europe, Asia, Far East Asia, Oceania, and Americas). For subjects who self-reported as Caucasian or white, the mean European factor score was 0.94 (median, 0.96) and the mean African factor score was 0.013 (median, 0.001). For subjects who self-reported as black, the mean African factor score was 0.74 (median, 0.77) and the mean European factor score was 0.23 (median, 0.20).

\section{Mouse Experiments}

Subjects. Alcohol-naive adult male and female Fkbp5 knockout (KO) and litter mate wild-type (WT) mice were used. These mice were generated through mating of heterozygous Fkbp5 mice as described in the previous report (Yong et al, 2007). KO mice used for this experiment have been backcrossed with C57BL/6J for four generations. On the first day of each experiment, mice were between 120 and 294 days old. During experiments, mice age matched for genotype were group-housed in polycarbonate cages $(29.2 \times 19.0 \times 12.7 \mathrm{~cm})$ with aspen wood shavings in groups of 2-4 per cage (experiment 1: acute alcohol exposure) or singly housed with nestlets, shredded paper, and chew toys (experiment 2: chronic alcohol exposure). Ambient room temperature was maintained at $21 \pm 2{ }^{\circ} \mathrm{C}$. Mice in experiment 1 had free access to food (Rodent Lab Diet 5001, Purina Mills, St Louis, MO) and water in the home cage at all times, except when testing procedures took place. Mice in experiment 2 had free access to a nutritionally complete liquid diet (Rodent Liquid Diet Lieber-DeCarli '82 Shake and Pour, Bio-Serv, Frenchtown, NJ), combined with up to $6 \%$ alcohol or calorie-matched maltodextrin, and water in the home cage at all times, except when testing procedures took place. Experimental procedures were conducted during the light phase of a 12:12 light:dark cycle (lights off at 1900 hours).

All experimental procedures were approved by the Purdue Animal Care and Use Committee and were conducted in accordance with the Guide for the Care and Use of Laboratory Animals.

Alcohol treatment. Alcohol (95\% v/v) was diluted to $20 \%$ $(\mathrm{v} / \mathrm{v})$ with $0.9 \%$ physiological saline and injected IP at a dose of $4.0 \mathrm{~g} / \mathrm{kg}(25.2 \mathrm{ml} / \mathrm{kg}$; experiment 1$)$ or diluted to $2,4.5$, or $6 \%(\mathrm{v} / \mathrm{v})$ in the nutritionally complete liquid diet (experiment 2).

Procedures. Handling-induced convulsion (HIC) procedures consisted of two baseline assessments conducted $20 \mathrm{~min}$ apart and immediately before alcohol/saline injections (experiment 1) or the introduction of alcohol into the liquid diet (experiment 2). HICs during withdrawal were assessed hourly beginning at $2 \mathrm{~h}$ after injection or removal of diet through hour 12 (6 measurements) and again at 24 and $25 \mathrm{~h}$ in withdrawal. Mice were gently and quickly lifted by the tail and spun $180^{\circ}$; the resultant convulsions were rated on a scale of severity ranging from 0 to 7 . The HICs technique and assessment scale were adapted from published methods of Crabbe and colleagues (e.g., Metten and Crabbe, 1994). Experimenters were blinded to genotype and treatment (alcohol vs control).

Baseline HICs were assessed at 0700 hours, immediately before the IP injections of alcohol or saline (experiment 1) or $48 \mathrm{~h}$ after the introduction of plain liquid diet and immediately before introduction of alcohol into the diet (experiment 2). HICs were then assessed hourly at 2-12, and again at 24 and $25 \mathrm{~h}$ following acute injection or following removal of the $6 \%$ alcohol liquid diet.

For experiment 1, 48 Fkbp5 KO (24 males and 24 females) and 43 WT (19 males and 24 females) mice were randomly assigned to receive acute exposure to either alcohol or saline; 5 Fkbp5 KO (2 males and 3 females) and 4 WT (0 males and 4 females) mice had previously undergone a similar HICs assessment procedure in which they had only received saline. For experiment 2, 8 Fkbp5 (3 males and 5 females) and 10 WT (4 males and 6 females) from experiment 1 were exposed to an alcohol liquid diet. Of these mice, approximately half had received alcohol injections and half had received saline injections previously, during experiment 1 . At least 4 months had passed since the end of experiment 1 and the start of experiment 2. For experiment 2, mice were gradually introduced to increasing percentages of alcohol over the course of 7 days, receiving 0 , 2 , and $4.5 \%$ alcohol diet for 2 days each until the final concentration of $6 \%$ was reached on day 7 . The $6 \%$ alcohol liquid diet was then supplied for 14 days, until day 21 when it was replaced with a calorie-matched maltodextrin liquid diet at 0700 hour for withdrawal assessment.

\section{Statistical Analysis}

We used the HaploView software program version 4.2 to assess HWE, LD (using the $\mathrm{D}^{\prime}$ and $r^{2}$ index), allelic/ haplotype frequencies, and haplotype blocks, the latter of which were defined by the default $\mathrm{D}^{\prime} / \mathrm{LOD}$ method (Barrett 
et al, 2005). The association analyses were conducted by linear regression using PLINK version 1.06 (Purcell, http://pngu.mgh.harvard.edu/ purcell/plink/) to calculate the effect size (beta coefficient) and significance level. The association of SNPs with withdrawal severity (maximum as well as average CIWA-Ar scores during withdrawal) was examined based on the most significant $p$-value among additive, allelic, dominant, and recessive model assumptions. The direction of the regression coefficient represents the effect of each extra minor allele. All models were also adjusted for age, gender, AIMs scores, pack-years of smoking, Fagerstrom scores, comorbidity with other substance dependence or not, use of other substances within the past 30 days, and the CPRS measures of depression and anxiety from day 2 , which may affect the alcohol withdrawal symptoms but have also been shown to be associated with FKBP5 variants (Binder et al, 2004, 2008, 2009; Xie et al, 2010). As BZD administration may also influence the withdrawal severity, we further adjusted the results for BZD usage (receiving BZD or not as well as total diazepam equivalent dose). To correct for multiple testing, we used the Benjamini-Hochberg method (Benjamini and Hochberg, 1995) to control for the false discovery rate (FDR). For haplotype-based analysis, the FDR represents a permutated $p$-value adjusted for testing multiple haplotypes (5000 permutations). In this study, we controlled the FDR at $q^{*}=0.05$ and assumed $p$-values were independently distributed under null hypotheses. A $p$-value $<0.05$ after multiple testing correction was considered to be statistically significant.

For mouse behavior analyses, HICs scores (baseline and withdrawal) were averaged based on scores from two independent raters with an historic inter-rater reliability of $>90 \%$. Baseline scores of KO and WT subjects were not significantly different; however, area under the curve (AUC) for all withdrawal time points $(2-12,24$, and $25 \mathrm{~h})$ was corrected with reference to the baseline score by subtracting each subject's average baseline score from each of their eight scores measured during the withdrawal time course (negative values were converted to a zero) (Metten and Crabbe, 1994). HICs data (AUC) were not normally distributed and so the non-parametric Kruskal-Wallis test was used for analysis. Probability values $<0.05$ were considered to be significant.

\section{RESULTS}

\section{Demographic Data}

Demographic and clinical characteristics of the 399 subjects (274 males and 125 females; mean age: $41.7 \pm 9.9$ ) are shown in Table 1.

\section{Single Marker Association}

The genotype information for each SNP is shown in Table 2. All six SNPs were significantly associated with both maximum (Table 3) and average (Supplementary Table 1) CIWA-Ar scores. Negative beta coefficients (rs3800373, rs9296158, rs1360780, and rs9470080) in the regression models indicate that individuals carrying the minor allele of these markers have lower CIWA-Ar scores, while positive
Table I Demographic and Clinical Characteristics of 399 Patients with Alcohol Dependence

\begin{tabular}{|c|c|}
\hline \multicolumn{2}{|l|}{ Sex } \\
\hline Male/female, N (\%) & $274 / 125(68.7 / 3 \mid .3)$ \\
\hline Age, mean (SD), years & $41.7(9.9)$ \\
\hline \multicolumn{2}{|l|}{ Race, N (\%) } \\
\hline EA & $230(57.6)$ \\
\hline AA & $136(34.1)$ \\
\hline Others & $33(8.3)$ \\
\hline Number of years of alcohol use, mean (SD) & $22.211(10.15)$ \\
\hline Number of years of intoxicating alcohol use, mean (SD) & $14.59(9.3)$ \\
\hline Alcohol dependence score, mean (SD) & $21.80(8.06)$ \\
\hline \multicolumn{2}{|l|}{ Timeline follow-back, 90 days, mean (SD) } \\
\hline Total drinks & $1164.12(751.53)$ \\
\hline Drinking days, days & $74.30(20.45)$ \\
\hline Average drinks/day, drinks & $15.23(8.23)$ \\
\hline Heavy drinking days, days & $70.32(23.88)$ \\
\hline Fagerstrom score, mean (SD) & $2.93(2.73)$ \\
\hline Cigarette pack-years, mean (SD) & $12.42(15.60)$ \\
\hline $\begin{array}{l}\text { With any diagnosis of other substance } \\
\text { dependence, } N(\%)^{\mathrm{a}}\end{array}$ & $140(35.1)$ \\
\hline Other substance use within the past 30 days, N (\%) & $234(58.7)$ \\
\hline Maximum scores on CIWA-Ar & $9.43(6.52)$ \\
\hline Average scores on CIWA-Ar & $6.48(5.21)$ \\
\hline \multicolumn{2}{|l|}{ Laboratory values } \\
\hline Albumin (U/I) & $4.10(0.36)$ \\
\hline GGT (g/dl) & | 82.65 (313.87) \\
\hline AST (U/l) & $68.96(71.84)$ \\
\hline ALT (U/I) & $63.09(54.08)$ \\
\hline ALP $(\cup / I)$ & $80.19(27.12)$ \\
\hline CDT (\%) & $0.09(0.09)$ \\
\hline Receiving BZD treatment, yes/no, N (\%) & $197(49.4) / 202(50.6)$ \\
\hline Diazepam equivalence dose (mg) & $83.26 \pm 110.37$ \\
\hline
\end{tabular}

Abbreviations: AA, African Americans; ALP, alkaline phosphatase; ALT, alanine transaminase; AST, aspartate transaminase; BZD, benzodiazepine; CDT, carbohydrate-deficient transferring; CIWA-Ar, Clinical Institute Withdrawal Assessment-Alcohol revised; EA, European Americans; GGT, gammaglutamyltransferase.

aOther substances include amphetamine, cocaine, cannabis, hallucinogen, inhalant, phencyclidine, sedatives, or opioids.

${ }^{b}$ Diazepam equivalent doses were logarithmically transformed for analysis.

coefficients (rs3777747 and rs9380524) indicate higher CIWA-Ar scores. All associations remained significant after adjusting for the potential covariates, receiving BZD or not, and total BZD dose. Even after further adjustment by $\mathrm{AD}$ severity scores and drinking variables from the TLFB data, all associations remained significant. When we tested the associations in European Americans only, we found rs3800373, rs9296158, rs1360780, and rs9470080 were significantly associated with withdrawal severity (see Supplementary Table 3). 
Table 2 The Genetic Information for FKBP5 Gene SNPs

\begin{tabular}{|c|c|c|c|c|c|c|}
\hline SNP & Chromosome position & Function & Alleles $1 / 2$ & Genotype distributions ( $1 \mathrm{I} / / 2 / 22)$ & MAF & HWE \\
\hline rs3800373 & 35650454 & $3^{\prime} \cup T R$ & $\mathrm{G} / \mathrm{T}$ & $44 / 166 / 184$ & 0.3223 & 0.49 \\
\hline rs9296158 & 35675060 & Intron 5 & $A / G$ & $51 / 165 / 182$ & 0.3354 & 0.18 \\
\hline rs3777747 & 35686980 & Intron 5 & $\mathrm{G} / \mathrm{A}$ & $83 / 195 / 119$ & 0.4547 & 0.84 \\
\hline rs9380524 & 35697048 & Intron 3 & $A / C$ & 1/64/332 & 0.0831 & 0.50 \\
\hline rs $\mid 360780$ & 35715549 & Intron 2 & $\mathrm{~T} / \mathrm{C}$ & $46 / 163 / 187$ & 0.3220 & 0.25 \\
\hline
\end{tabular}

Abbreviations: HWE, p-values of the Hardy-Weinberg equilibrium; MAF, minor allele frequency.

Alleles I and 2 refer to minor and major allele, respectively. Genotype distributions are shown as the counts of three genotypes, II/I2/22.

Table 3 Associations between FKBP5 Gene SNPs and Alcohol Withdrawal Severity Measured by CIWA-Ar

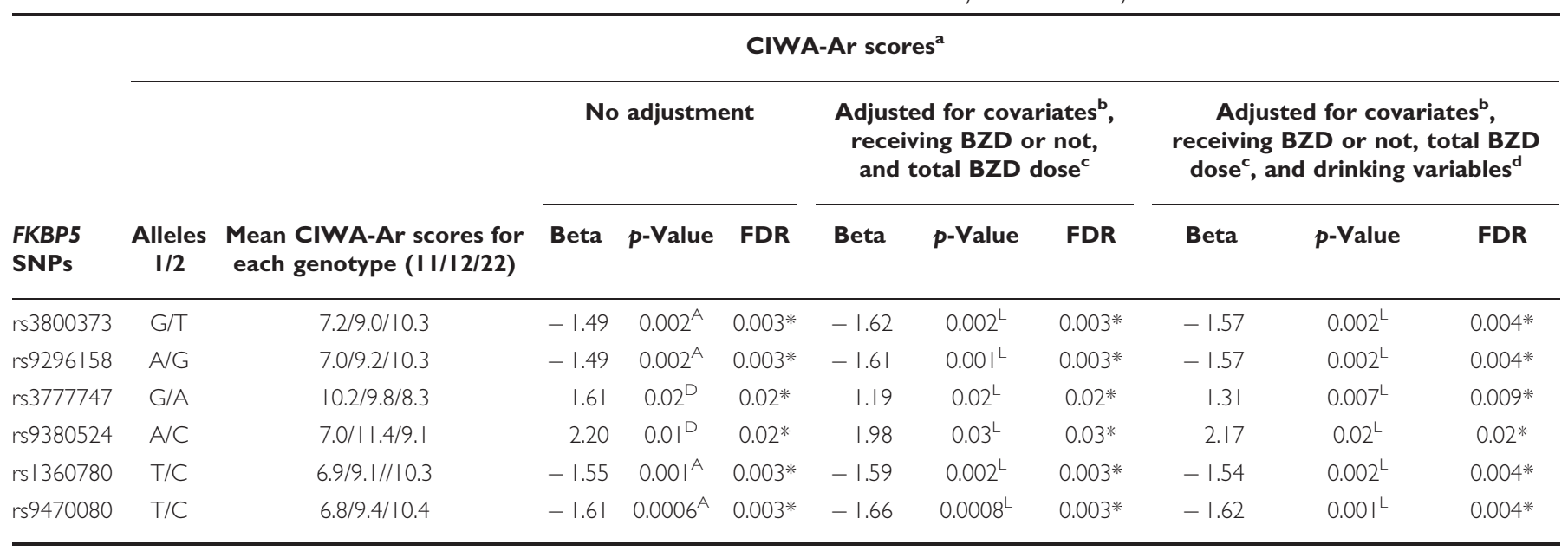

Abbreviations: ADS, Alcohol Dependence Scale; AlMs, ancestry informative markers; BZD, benzodiazepine; CIWA-Ar, Clinical Institute Withdrawal AssessmentAlcohol revised; CPRS, Comprehensive Psychopathological Rating Scale; FDR, false discovery rate; TLFB, timeline follow-back.

Alleles I and 2 refer to minor and major allele, respectively.

$P$-value represents the minimal $p$-value from four models, including additive $(A)$, allelic $(L)$, dominant $(D)$, and recessive $(R)$ models.

FDR was calculated using Benjamini and Hochberg's method (1995) for multiple testing corrections.

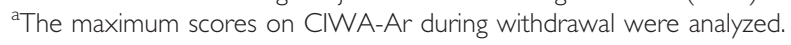

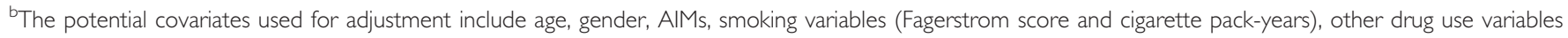
(comorbidity with other drug dependence and drug use within the past 30 days), baseline depression and anxiety scores on CPRS.

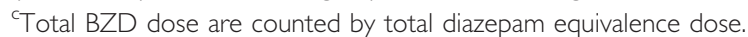

dDrinking variables include ADS scores and TLFB data (TLFB measurement of alcohol consumption variables during the preceding 3 months including total drinks, number of drinking days, average drinks per day, and heavy drinking days).

* $p<0.05$.

\section{LD Structure}

Figure 1 shows that the six SNPs that span FKBP5 are in strong LD. There are four haplotypes with frequency $>5 \%$ accounting for $94.5 \%$ of the haplotype diversity. We compared the structures of LD across the 150-kb region containing the FKBP5 gene using genotype data from the CEU and ASW HapMap samples and found the LD structure across this region showed high similarity between the two populations.

\section{Haplotype-Based Association}

We examined the association of haplotypes based on the selected SNPs with overall maximum (Table 4) and average (Supplementary Table 2) withdrawal severity. Haplotype
GAACTT (frequency $=0.30$ ) and the complementary haplotype TGGACC (frequency $=0.08$ ) were associated with lower and higher withdrawal severity, respectively. These results were consistent with what we found using the individual markers, that is, haplotype GAACTT was composed of alleles on each maker that were associated with lower CIWA-Ar scores, whereas TGGACC included alleles that were associated with higher CIWA-Ar scores (see Table 3). Associations for the two haplotypes, however, were no longer significant after adjustment for covariates. In the subsample of European Americans, the significant association between haplotype GAACTT and alcohol withdrawal severity also disappeared after covariate adjustment (Supplementary Table 4). When we examined haplotypes composed of rs3800373, rs9296158, rs1360780, and rs9470080 (see Supplementary Figure 1 for LD structure of these four SNPs), which have been previously 


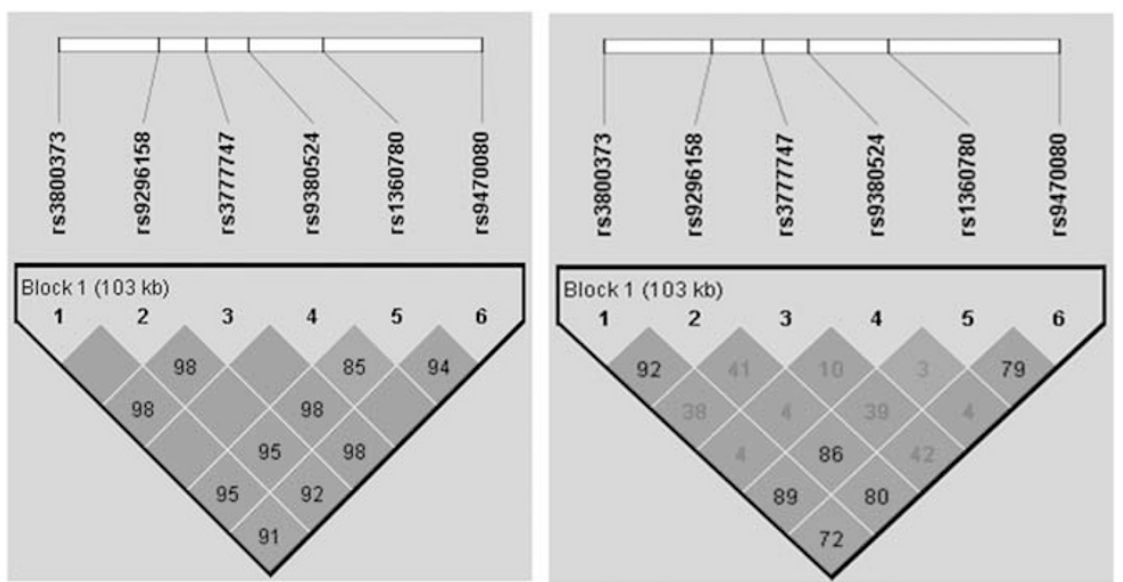

Figure I The haplotype structure of six FKBP5 gene SNPs. The numbers in the squares refer to pairwise LD measured as D' (left) and $r^{2}$ (right).

Table 4 Haplotype-Based Association of FKBP5 Gene SNPs with Alcohol Withdrawal Severity Measured by CIWA-Ar

CIWA-Ar scores ${ }^{\mathrm{a}}$

\begin{tabular}{|c|c|c|c|c|c|c|c|c|c|c|}
\hline Haplotype & Frequency & \multicolumn{3}{|c|}{ No adjustment } & \multicolumn{3}{|c|}{$\begin{array}{l}\text { Adjusted for covariates } \\
\text { receiving } B Z D \text { or not, } \\
\text { and total BZD dose }{ }^{c}\end{array}$} & \multicolumn{3}{|c|}{$\begin{array}{l}\text { Adjusted for covariates }{ }^{b} \text {, receiving } \\
\text { BZD or not, total BZD dose } \\
\text { and drinking variables }\end{array}$} \\
\hline \multicolumn{11}{|c|}{ FKBP5 gene (rs3800373-rs9296I58-rs3777747-rs9380524-rs|360780-rs9470080) } \\
\hline GAACTT & 0.2990 & -1.49 & 0.002 & $0.007^{*}$ & -0.54 & 0.14 & 0.42 & -0.30 & 0.40 & 0.82 \\
\hline TGGCCC & 0.3680 & 0.53 & 0.27 & 0.66 & 0.20 & 0.57 & 0.95 & 0.32 & 0.36 & 0.78 \\
\hline TGACCC & 0.1830 & 0.70 & 0.21 & 0.57 & 0.40 & 0.34 & 0.75 & 0.12 & 0.78 & 0.99 \\
\hline
\end{tabular}

Abbreviations: ADS, Alcohol Dependence Scale; AlMs, ancestry informative markers; BZD, benzodiazepine; CIWA-Ar, Clinical Institute Withdrawal AssessmentAlcohol revised; CPRS, Comprehensive Psychopathological Rating Scale; FDR, false discovery rate; TLFB, timeline follow-back.

Results were based on haplotype-based association tests; only haplotypes with frequency $>0.05$ were considered.

FDR represents a permutated p-value adjusted for testing multiple haplotypes (5000 permutations).

${ }^{\text {aT }}$ The maximum scores on CIWA-Ar during withdrawal were analyzed.

'The potential covariates used for adjustment include age, gender, AIMs, smoking variables (Fagerstrom score and cigarette pack-years), other drug use variables (comorbidity with other drug dependence and drug use within the past 30 days), baseline depression and anxiety scores on CPRS.

${ }^{c}$ Total BZD dose are counted by total diazepam equivalence dose.

${ }^{\mathrm{d} D}$ rinking variables include ADS scores and TLFB data (TLFB measurement of alcohol consumption variables during the preceding 3 months including total drinks, number of drinking days, average drinks per day, and heavy drinking days)

* $p<0.05$.

reported to be potentially functional and associated with several measures of psychopathology (Binder et al, 2004, 2008; Ising et al, 2008; Roy et al, 2010; Xie et al, 2010), we found that haplotype GATT and the complementary TGCC were positively and negatively associated with withdrawal severity, respectively (Supplementary Table 5); however, these results also did not remain after adjustment for covariates. These findings suggest that haplotypes consisting of minor alleles are linked to a lower severity of alcohol withdrawal.

\section{HICs in Fkbp5 KO and WT Mice following Acute Alcohol Exposure}

Analysis of treatment group AUC within each genotype using the Kruskal-Wallis non-parametric test showed a main effect of treatment group in $\mathrm{KO}$ mice $(\mathrm{H}(1)=4.859$, $p=0.028$; alcohol $>$ saline) but not in WT mice (Figure 2).

\section{HICs Fkbp5 KO and WT Mice following Chronic Alcohol Exposure}

Analysis of HICs AUC using the Kruskal-Wallis non-parametric test showed a main effect of genotype $(\mathrm{H}(1)=4.381$, $p=0.036 ; \mathrm{KO}>\mathrm{WT}$ ) (Figure 3).

\section{DISCUSSION}

We found that FKBP5 gene SNPs (rs3800373, rs9296158, rs3777747, rs9380524, rs1360780, and rs9470080), both 

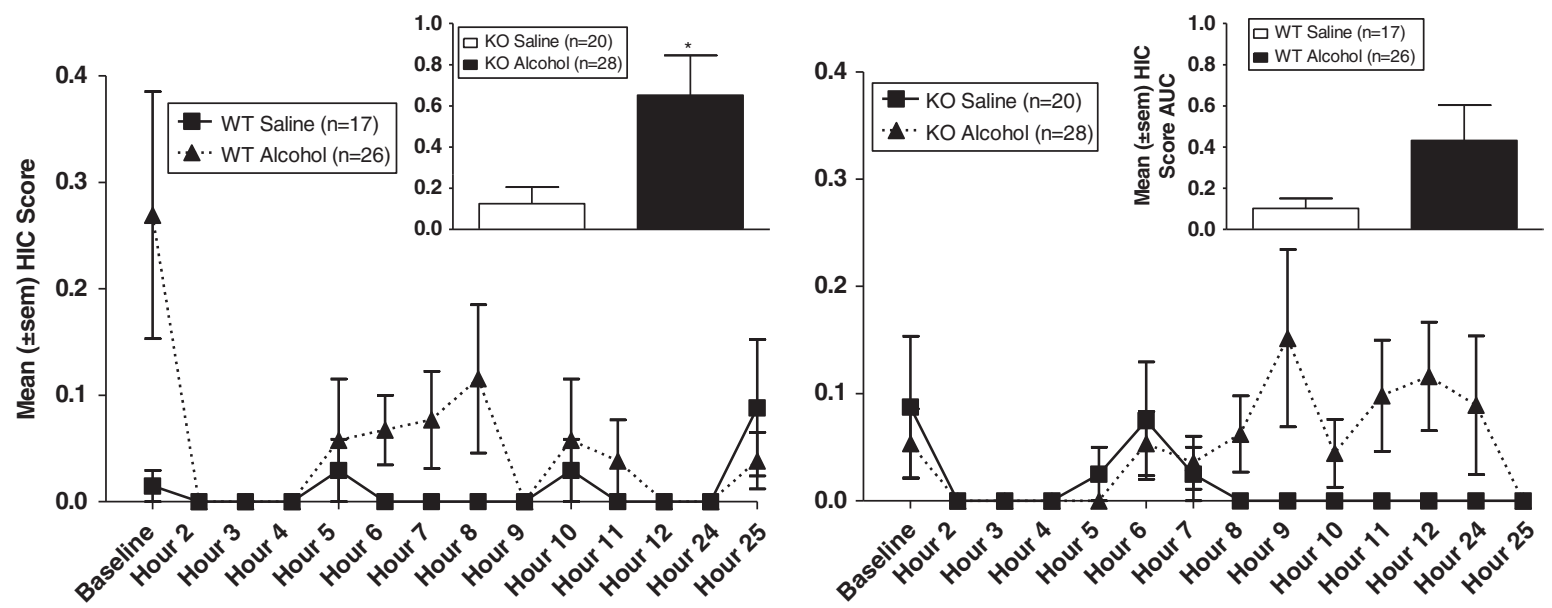

Figure 2 Mean ( \pm SEM) HICs at each time point (baseline, 2-12, 24, and $25 \mathrm{~h}$ ) following acute injection of either alcohol or saline. Inset: mean ( \pm SEM) AUC (corrected for baseline) for HICs across the withdrawal measurement time course. ${ }^{*} p<0.05$; alcohol $>$ saline.

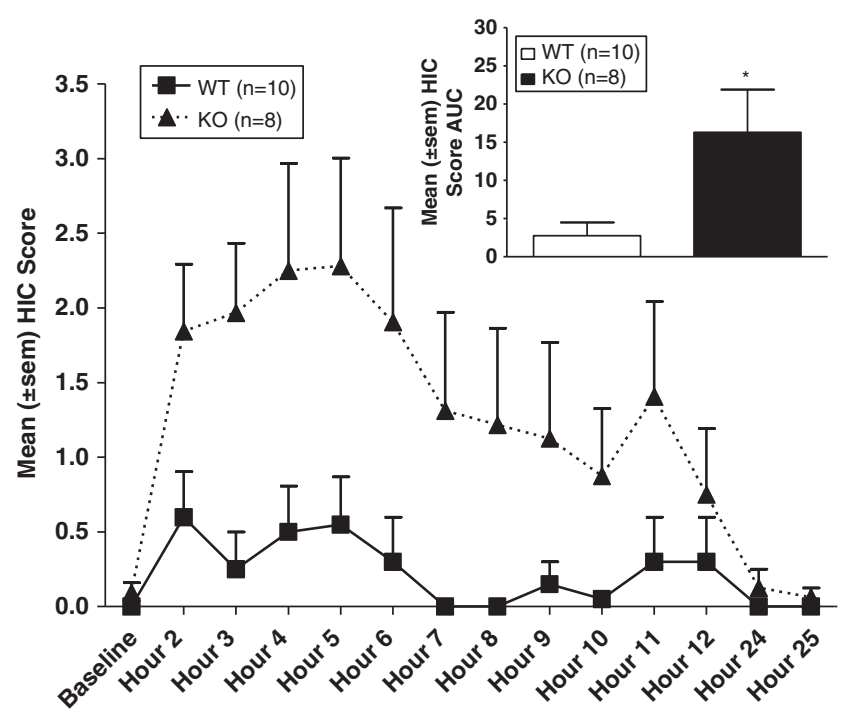

Figure 3 Mean $( \pm$ SEM) HICs at each time point (baseline, 2-12, 24, and $25 \mathrm{~h}$ ) following removal of alcohol diet. Inset: mean ( \pm SEM) AUC (corrected for baseline) for HICs across the withdrawal measurement time course. ${ }^{*} p<0.05, \mathrm{KO}>\mathrm{WT}$.

individually and in a haplotype-based analysis, influence the severity of alcohol withdrawal severity. To our knowledge, this is the first study to investigate the potential genetic factors influencing severity of the overall AWS, as previous studies of predisposing characteristics for severe alcohol withdrawal were focused on DT, seizures, or hallucinations. As human genetic association studies are fraught with numerous potential confounds and frequently fail to replicate, we validated our findings using genetically modified mice. We found converging evidence in a KO mouse model that suggests an important role for the Fkbp5 gene in modulating alcohol withdrawal severity. KO mice showed greater alcohol withdrawal-induced HICs following chronic alcohol exposure compared with WT mice.

The SNPs in the FKBP5 gene with possible functional effects (eg, rs1360780) have been suggested to serve as 'intronic enhancers,' which are intronic SNPs with a binding site for transcriptional factors such as GR (Binder, 2009). Upon cortisol binding, a direct GR-mediated upregulation of FKBP5 expression via the intronic response elements (Vermeer et al, 2003) will act as part of an intracellular ultra-short negative feedback loop for GR activity, conferring a state of GR resistance and affecting HPA axis responsivity. Several FKBP5 gene variants have been demonstrated to be functionally involved in the regulation of HPA axis responsivity. For example, in vitro exposure of cells to glucocorticoids induces an increase in FKBP5 mRNA, with genotypes composed of rs 1360780 and rs3800373 minor alleles being the highest expressing variants (Vermeer et al, 2003). In humans, healthy adults homozygous for these alleles show a greater and more prolonged cortisol response during the Trier Social Stress Test compared with those who have complementary genotypes (Ising et al, 2008). These SNPs, as well as rs9296158 and rs9470080, have been associated with a higher FKBP5 expression and a stronger induction of FKBP5 mRNA by cortisol (Binder et al, 2004; Tatro et al, 2010), and subsequently have been correlated with depression (Binder et al, 2004; Tatro et al, 2010) and suicide (Brent et al, 2010; Perez-Ortiz et al, 2013). They also affect cortisol responses and result in diminished dexamethasone suppression (Binder et al, 2008). In addition, the minor alleles of rs9470080 and rs1360780 are associated with total cortisol secretion during the day expressed as the AUC (cortisol ${ }_{\mathrm{AUC}}$ ) (Velders et al, 2011). Finally, a haplotype of the above-listed SNPs has been associated with suicidal attempts (Roy et al, 2010) or aggressive behavior (Bevilacqua et al, 2012) among individuals with childhood trauma.

The functional association between FKBP5 gene variants and HPA axis regulation appears to be altered, for example, the high expression alleles may instead be associated with increased GR sensitivity, in the presence of disease where feedback inhibition has been impaired along with activation of the HPA axis (Binder et al, 2004; Bevilacqua et al, 2012). Such is the case in subjects with depression or PTSD, in whom the high expression alleles are associated with greater glucocorticoid sensitivity, compared with controls where they are associated with increased glucocorticoid resistance 
(Binder et al, 2004, 2008; Bevilacqua et al, 2012). Binder et al (2004, 2009) suggest that the high expression alleles of the FKBP5 gene may counteract depression-related HPA axis hyperactivity via compensatory mechanisms, allowing a faster restoration of normal HPA axis function and a more rapid response to antidepressants in depressed individuals. It is possible that similar mechanisms may also have a role in the reduced AWS severity we observed in minor allele carriers of the FKBP5 variants. Given that AWS also produces a pattern of HPA axis dysregulation, and a normalization of HPA axis responsivity has been associated with resolution of AWS (Costa et al, 1996; Adinoff et al, 1998, 2005; Esel et al, 2001) individuals with higher expression alleles (eg, rs3800373, rs92962158, rs1360780, and rs9470080) may have better compensatory adaptive mechanisms to resist AWS, and thus manifest lower CIWAAr scores. Although the in vitro function and biological impact of rs3777747 and rs9380524 has not been studied yet, we postulate that the minor alleles of these two SNPs ( $G$ and A allele, respectively) are not high expression alleles favoring compensatory mechanisms, because of the observation that the minor allele carriers manifest higher CIWA-Ar scores, suggesting a poorer adaptive response to HPA activation. Our haplotype analyses also showed a consistent pattern of association between minor alleles of each SNP and withdrawal severity, that is, two complementary haplotypes involving the SNPs (GAACTT and TGGACC) were associated with lower and higher CIWA-Ar scores, respectively, although the association disappeared after adjustment for covariates. In sum, our finding adds to existing evidence that genetic factors contribute to the central neuroadaptation to alcohol withdrawal-induced impairment of HPA axis regulation in humans. Our findings of greater withdrawal after chronic alcohol exposure in $\mathrm{KO}$ mice are in agreement with the notion that FKBP5 function affects alcohol withdrawal severity.

Findings from this study should be interpreted in light of several limitations. Previous investigations of the genetic component of alcohol withdrawal-related phenotypes have typically classified subjects as with or without a specific history of DTs, hallucinations, and seizures (Schmidt and Sander, 2000; Koehnke et al, 2002; Okubo et al, 2003; Schumann et al, 2003; Wernicke et al, 2003; Limosin et al, 2004; Rujescu et al, 2005; Tadic et al, 2005; Preuss et al, 2006; van Munster et al, 2007; Karpyak et al, 2010; Du et al, 2011). It is important to note that the withdrawal severity in our patients was generally mild to moderate, and as a result our findings cannot be extended to patients exhibiting severe forms of withdrawal. Similarly, the HIC measure in mice assesses physical withdrawal signs that vary on a spectrum of mild to severe (convulsion-like signs are rated on a scale of $0-7$ ) and the degree of withdrawal severity observed in this study was also mild to moderate in magnitude. Moreover, HIC measurement is but one way to assess physical withdrawal severity. As a result, additional detailed methods to assess withdrawal severity as well as the inclusion of more severe forms of withdrawal phenotypes should be considered. Furthermore, HIC measurement can only be used in mice. There is likely an incomplete overlap of the mechanisms regulating the withdrawal phenotypes measured between mice and humans. Second, it is not clear whether the associations between FKBP5 SNPs and withdrawal severity may be attributed to other comorbid psychiatric disorders, such as major depression or PTSD. In addition, childhood trauma has been shown to interact with FKBP5 gene variation to influence the stress response (Binder et al, 2008; Roy et al, 2010; Xie et al, 2010; Bevilacqua et al, 2012) and is considered to be a confounding variable in HPA axis dysregulation in alcohol-dependent patients (Schafer et al, 2010). Therefore, we were unable to exclude the contribution from stressful life events to our results. Third, the haplotype-based findings may be biased, in that the SNPs in our study were selected from those that have been published previously rather than based on independent criteria for tag SNP selection across the entire gene locus. We do not know whether the results would be different after examination of additional SNPs. Fourth, we did not include heterozygous animals that may provide insight into the recessive and dominant characteristics of Fkbp5 gene and the pertinent underlying mechanisms. Fifth, data on cortisol/coticosterone levels or HPA axis reactivity were not available for our human subjects or for the mice. Therefore, we are not able to directly assess potential moderating effects of FKBP5 SNPs on HPA axis dysregulation associated with AWS. We believe direct endocrine measures including $\mathrm{CRH}$ levels, which is also strongly implicated in the pathogenesis of $\mathrm{AD}$ (Becker, 2012), are needed in future studies to elucidate the roles of FKBP5 in regulating HPA axis activity, GR sensitivity, and the feedback on CRH. Sixth, it should be noted that there are other pathways, other than regulation of the GR, by which FKBP5 may influence the alcohol withdrawal phenomenon. For instance, FKBP5 has direct anxiogenic effects when overexpressed in the amygdala (Attwood et al, 2011). Moreover, FKBP5 and indirect GR sensitivity could feedback on CRH levels, which may be directly involved in the mediation of AWS (Sommer et al, 2008). Last but not least, while the Fkbp KO mouse experiments support the role of FKBP5 gene involvement in alcohol withdrawal, they do not explicitly model human genetic variation. In addition, possible compensatory changes that occur during development may have been overlooked in this study. The utilization of different types of genetic animal models such as a congenic mouse model to explore the role of genetic variation at genetic loci is warranted. Despite these limitations, our findings suggest that a potential role for FKBP5 gene in modulating alcohol withdrawal severity is an important avenue of research that warrants further study, in particular, mechanistic exploration of the functional significance of the SNP variants and their association with the alcohol withdrawal phenotypes studied here.

In conclusion, we show that the FKBP5 gene in mice and FKBP5 genetic variants in humans are significantly associated with alcohol withdrawal severity. These differences in withdrawal severity outcome may be attributable to variation in the ability of these variants to regulate FKBP5 expression (Binder et al, 2004, 2009) and ensuing HPA axis responsivity. Further studies are needed to replicate and verify these tentative allelic associations.

\section{FUNDING AND DISCLOSURE}

The authors declare no conflict of interest. 


\section{ACKNOWLEDGEMENTS}

We thank Matthew S Powers, Kristen R Breit, Monte Phillips, Hui Sun, and Judy Powers for providing technical assistance on this project. We also express our appreciation to Drs Weinian Shou and Weidong Yong for their support on animal breeding and valuable discussions over this research.

\section{REFERENCES}

Adinoff B, Iranmanesh A, Veldhuis J, Fisher L (1998). Disturbances of the stress response: the role of the HPA axis during alcohol withdrawal and abstinence. Alcohol Health Res World 22: 67-72.

Adinoff B, Junghanns K, Kiefer F, Krishnan-Sarin S (2005). Suppression of the HPA axis stress-response: implications for relapse. Alcohol Clin Exp Res 29: 1351-1355.

Asberg M, Montgomery SA, Perris C, Schalling D, Sedvall G (1978). A comprehensive psychopathological rating scale. Acta Psychiatr Scand Suppl 271: 5-27.

Attwood BK, Bourgognon JM, Patel S, Mucha M, Schiavon E, Skrzypiec AE et al (2011). Neuropsin cleaves EphB2 in the amygdala to control anxiety. Nature 473: 372-375.

Barrett JC, Fry B, Maller J, Daly MJ (2005). Haploview: analysis and visualization of LD and haplotype maps. Bioinformatics 21: 263-265.

Becker HC (2012). Effects of alcohol dependence and withdrawal on stress responsiveness and alcohol consumption. Alcohol Res 34: 448-458.

Benjamini Y, Hochberg Y (1995). Controlling the false discovery rate: a practical and powerful approach to multiple testing. $J$ R Stat Soc Series B (Methodological) 57: 289-300.

Bevilacqua L, Carli V, Sarchiapone M, George DK, Goldman D, Roy A et al (2012). Interaction between FKBP5 and childhood trauma and risk of aggressive behavior. Arch Gen Psychiatry 69: 62-70.

Binder EB (2009). The role of FKBP5, a co-chaperone of the glucocorticoid receptor in the pathogenesis and therapy of affective and anxiety disorders. Psychoneuroendocrinology 34(Suppl 1): S186-S195.

Binder EB, Bradley RG, Liu W, Epstein MP, Deveau TC, Mercer KB et al (2008). Association of FKBP5 polymorphisms and childhood abuse with risk of posttraumatic stress disorder symptoms in adults. JAMA 299: 1291-1305.

Binder EB, Kunzel HE, Nickel T, Kern N, Pfennig A, Majer M et al (2009). HPA-axis regulation at in-patient admission is associated with antidepressant therapy outcome in male but not in female depressed patients. Psychoneuroendocrinology 34: 99-109.

Binder EB, Salyakina D, Lichtner P, Wochnik GM, Ising M, Putz B et al (2004). Polymorphisms in FKBP5 are associated with increased recurrence of depressive episodes and rapid response to antidepressant treatment. Nat Genet 36: 1319-1325.

Brent D, Melhem N, Ferrell R, Emslie G, Wagner KD, Ryan N et al (2010). Association of FKBP5 polymorphisms with suicidal events in the Treatment of Resistant Depression in Adolescents (TORDIA) study. Am J Psychiatry 167: 190-197.

Costa A, Bono G, Martignoni E, Merlo P, Sances G, Nappi G (1996). An assessment of hypothalamo-pituitary-adrenal axis functioning in non-depressed, early abstinent alcoholics. Psychoneuroendocrinology 21: 263-275.

Du Y, Nie Y, Li Y, Wan YJ (2011). The association between the SLC6A3 VNTR 9-repeat allele and alcoholism-A meta-analysis. Alcohol Clin Exp Res 35: 1625-1634.

Esel E, Sofuoglu S, Aslan SS, Kula M, Yabanoglu I, Turan MT (2001). Plasma levels of beta-endorphin, adrenocorticotropic hormone and cortisol during early and late alcohol withdrawal. Alcohol Alcohol 36: 572-576.
First MB, Spitzer RL, Gibbon M, Williams JBW (1996): Structured Clinical Interview for DSM-IV Axis I Disorders (SCID-I), Clinician Version. American Psychiatric Press: Washington, DC.

Heilig M, Egli M, Crabbe JC, Becker HC (2010). Acute withdrawal, protracted abstinence and negative affect in alcoholism: are they linked? Addict Biol 15: 169-184.

Hodgkinson CA, Yuan Q, Xu K, Shen PH, Heinz E, Lobos EA et al (2008). Addictions biology: haplotype-based analysis for 130 candidate genes on a single array. Alcohol Alcohol 43: 505-515.

Ising M, Depping AM, Siebertz A, Lucae S, Unschuld PG, Kloiber S et al (2008). Polymorphisms in the FKBP5 gene region modulate recovery from psychosocial stress in healthy controls. Eur $\mathrm{J}$ Neurosci 28: 389-398.

Karpyak VM, Biernacka JM, Weg MW, Stevens SR, Cunningham JM, Mrazek DA et al (2010). Interaction of SLC6A4 and DRD2 polymorphisms is associated with a history of delirium tremens. Addict Biol 15: 23-34.

Karpyak VM, Kim JH, Biernacka JM, Wieben ED, Mrazek DA, Black JL et al (2009). Sequence variations of the human MPDZ gene and association with alcoholism in subjects with European ancestry. Alcohol Clin Exp Res 33: 712-721.

Kendler KS, Aggen SH, Prescott CA, Crabbe J, Neale MC (2012). Evidence for multiple genetic factors underlying the DSM-IV criteria for alcohol dependence. Mol Psychiatry 17: 1306-1315.

Klengel T, Mehta D, Anacker C, Rex-Haffner M, Pruessner JC, Pariante CM et al (2013). Allele-specific FKBP5 DNA demethylation mediates gene-childhood trauma interactions. Nat Neurosci 16: 33-41.

Koehnke MD, Schick S, Lutz U, Willecke M, Koehnke AM, Kolb W et al (2002). Severity of alcohol withdrawal symptoms and the T1128C polymorphism of the neuropeptide Y gene. J Neural Transm 109: 1423-1429.

Limosin F, Loze JY, Boni C, Fedeli LP, Hamon M, Rouillon F et al (2004). The A9 allele of the dopamine transporter gene increases the risk of visual hallucinations during alcohol withdrawal in alcohol-dependent women. Neurosci Lett 362: 91-94.

McClintick JN, Xuei X, Tischfield JA, Goate A, Foroud T, Wetherill L et al (2013). Stress-response pathways are altered in the hippocampus of chronic alcoholics. Alcohol 47: 505-515.

Metten P, Crabbe JC (1994). Common genetic determinants of severity of acute withdrawal from ethanol, pentobarbital and diazepam in inbred mice. Behav Pharmacol 5: 533-547.

Metten P, Crabbe JC (2005). Alcohol withdrawal severity in inbred mouse (Mus musculus) strains. Behav Neurosci 119: 911-925.

Okubo T, Harada S, Higuchi S, Matsushita S (2003). Association analyses between polymorphisms of the phase II detoxification enzymes (GSTM1, NQO1, NQO2) and alcohol withdrawal symptoms. Alcohol Clin Exp Res 27: 68S-71S.

Perez-Ortiz JM, Garcia-Gutierrez MS, Navarrete F, Giner S, Manzanares J (2013). Gene and protein alterations of FKBP5 and glucocorticoid receptor in the amygdala of suicide victims. Psychoneuroendocrinology 38: 1251-1258.

Preuss UW, Zill P, Koller G, Bondy B, Hesselbrock V, Soyka M (2006). Ionotropic glutamate receptor gene GRIK3 SER310ALA functional polymorphism is related to delirium tremens in alcoholics. Pharmacogenomics J 6: 34-41.

Roberts AJ, Crabbe JC, Keith LD (1994). Corticosterone increases severity of acute withdrawal from ethanol, pentobarbital, and diazepam in mice. Psychopharmacology 115: 278-284.

Rose AK, Shaw SG, Prendergast MA, Little HJ (2010). The importance of glucocorticoids in alcohol dependence and neurotoxicity. Alcohol Clin Exp Res 34: 2011-2018.

Roy A, Gorodetsky E, Yuan Q, Goldman D, Enoch MA (2010). Interaction of FKBP5, a stress-related gene, with childhood trauma increases the risk for attempting suicide. Neuropsychopharmacology 35: 1674-1683.

Rujescu D, Soyka M, Dahmen N, Preuss U, Hartmann AM, Giegling I et al (2005). GRIN1 locus may modify the susceptibility 
to seizures during alcohol withdrawal. Am J Med Genet B Neuropsychiatr Genet 133B: 85-87.

Schafer I, Teske L, Schulze-Thusing J, Homann K, Reimer J, Haasen C et al (2010). Impact of childhood trauma on hypothalamus-pituitary-adrenal axis activity in alcoholdependent patients. Eur Addict Res 16: 108-114.

Schmidt LG, Sander T (2000). Genetics of alcohol withdrawal. Eur Psychiatry 15: 135-139.

Schumann G, Rujescu D, Kissling C, Soyka M, Dahmen N, Preuss UW et al (2003). Analysis of genetic variations of protein tyrosine kinase fyn and their association with alcohol dependence in two independent cohorts. Biol Psychiatry 54: 1422-1426.

Sharrett-Field L, Butler TR, Berry JN, Reynolds AR, Prendergast MA (2013). Mifepristone pretreatment reduces ethanol withdrawal severity in vivo. Alcohol Clin Exp Res 37: 1417-1423.

Shirley RL, Walter NA, Reilly MT, Fehr C, Buck KJ (2004). Mpdz is a quantitative trait gene for drug withdrawal seizures. Nat Neurosci 7: 699-700.

Skinner H, Horn J (1984): Alcohol Dependence Scale: User's Guide. Addiction Research Foundation: Toronto, Canada.

Sommer WH, Rimondini R, Hansson AC, Hipskind PA, Gehlert DR, Barr CS et al (2008). Upregulation of voluntary alcohol intake, behavioral sensitivity to stress, and amygdala crhr1 expression following a history of dependence. Biol Psychiatry 63: 139-145.

Stephens MA, Wand G (2012). Stress and the HPA axis: role of glucocorticoids in alcohol dependence. Alcohol Res 34: 468-483.

Tadic A, Dahmen N, Szegedi A, Rujescu D, Giegling I, Koller G et al (2005). Polymorphisms in the NMDA subunit 2B are not associated with alcohol dependence and alcohol withdrawalinduced seizures and delirium tremens. Eur Arch Psychiatry Clin Neurosci 255: 129-135.

Tatro ET, Nguyen TB, Bousman CA, Masliah E, Grant I, Atkinson $\mathrm{JH}$ et al (2010). Correlation of major depressive disorder symptoms with FKBP5 but not FKBP4 expression in human immunodeficiency virus-infected individuals. I Neurovirol 16: 399-404. van Munster BC, Korevaar JC, de Rooij SE, Levi M, Zwinderman AH (2007). Genetic polymorphisms related to delirium tremens: a systematic review. Alcohol Clin Exp Res 31: 177-184.

Velders FP, Kuningas M, Kumari M, Dekker MJ, Uitterlinden AG, Kirschbaum C et al (2011). Genetics of cortisol secretion and depressive symptoms: a candidate gene and genome wide association approach. Psychoneuroendocrinology 36: 1053-1061.

Vendruscolo LF, Barbier E, Schlosburg JE, Misra KK, Whitfield TW Jr., Logrip ML et al (2012). Corticosteroid-dependent plasticity mediates compulsive alcohol drinking in rats. J Neurosci 32: 7563-7571.

Vermeer H, Hendriks-Stegeman BI, van der Burg B, van BuulOffers SC, Jansen M (2003). Glucocorticoid-induced increase in lymphocytic FKBP51 messenger ribonucleic acid expression: a potential marker for glucocorticoid sensitivity, potency, and bioavailability. J Clin Endocrinol Metab 88: 277-284.

Wernicke C, Samochowiec J, Schmidt LG, Winterer G, Smolka M, Kucharska-Mazur J et al (2003). Polymorphisms in the N-methyl$\mathrm{D}$-aspartate receptor 1 and $2 \mathrm{~B}$ subunits are associated with alcoholism-related traits. Biol Psychiatry 54: 922-928.

Wochnik GM, Ruegg J, Abel GA, Schmidt U, Holsboer F, Rein T (2005). FK506-binding proteins 51 and 52 differentially regulate dynein interaction and nuclear translocation of the glucocorticoid receptor in mammalian cells. J Biol Chem 280: 4609-4616.

Xie P, Kranzler HR, Poling J, Stein MB, Anton RF, Farrer LA et al (2010). Interaction of FKBP5 with childhood adversity on risk for post-traumatic stress disorder. Neuropsychopharmacology 35: 1684-1692.

Yong W, Yang Z, Periyasamy S, Chen H, Yucel S, Li W et al (2007). Essential role for Co-chaperone Fkbp52 but not Fkbp51 in androgen receptor-mediated signaling and physiology. J Biol Chem 282: 5026-5036.

Zannas AS, Binder EB (2014). Gene-environment interactions at the FKBP5 locus: sensitive periods, mechanisms and pleiotropism. Genes Brain Behav 13: 25-37.

Supplementary Information accompanies the paper on the Neuropsychopharmacology website (http://www.nature.com/npp) 